\title{
3D Laser Microscopy for Nanotechnology and Metrology
}

\author{
S. Yatsunenko*, M. Fabich \\ Institute of Physics, Polish Academy of Sciences, al. Lotników 32/46, 02-668 Warsaw, Poland \\ and \\ Olympus Europa - http://www.olympus-europa.com/
}

In this article we would like to present new LEXT OLS4000 — 3D measuring laser microscope for material science with an example of application - structural investigations of antibacterial textile (material was passivated with silver nanoparticles).

\section{Introduction}

Nanosized silver particles open way to new breed of antibacterial (antimicrobial) materials. Silver particles could be now incorporated into polypropylene to produce an antibacterial material that could be used in anything from carpets, to napkins and surgical masks.

Silver has been medically proven to kill over 650 disease-causing organisms in the body and is also very safe. By combining silver and polypropylene to produce an organic-inorganic fibre, researchers have produced the first safe, antibacterial fibre with a wide range of possible applications.

Polypropylene fibre is one of the most widely used synthetic fibres in the textile industry because it is cheaper and stronger than other synthetic fibres. Researchers have demonstrated, in a paper to be published in the July issue of Polymer International, available online in June, that they can produce two types of fibres both with silver particles distributed evenly along the length of the fibre.

According to investigations, used nanosized silver particles maximize the surface area and give the optimum antibacterial effect. Fibres containing silver in the core part had no antimicrobial activity. However, the fibres that included silver in the sheath part showed excellent antibacterial effect.

That is why very important challenge is to detect silver nanoparticles deposition on textile material.

One of the main question related to the textile material with deposited silver nanoparticles is which method could be used for quick, nondestructive and comprehensive control of silver nanoparticles deposition.

Briefly, structure investigations of "nano-silver" textile material made with help of Olympus LEXT OLS4000 are presented below.

\footnotetext{
* e-mail: yatsun@ifpan.edu.pl
}

\section{Samples}

Two textile materials with silver nanoparticles (fibres should be covered by silver agglomerates with characteristic nanosizes) and without it.

\section{The main goal of experiment}

Using confocal method detect presence and location of silver nanoparticles on textile, visualize and analyze size of silver nanoparticles.

\section{Observations conditions}

New LEXT OLS4000 — 3D measuring laser microscope for material science made by Olympus was used for structural investigation and inspections of textile samples with silver nanoparticles and without it.

Simultaneous acquisition of brightness, height, and color information in the same visual field with high-resolution observation with high-accuracy, non-contact measurement at near-nanometer precision without sample preparation were used. There are several objectives which could be used for investigations, such as: $2.5 \times$, $5 \times, 10 \times, 20 \times, 50 \times, 100 \times$ and $150 \times$ which give magnification range from $108 \times$ to $17200 \times$. Using UV laser diode with $405 \mathrm{~nm}$ excitation wavelength and two photomultiplier detectors with double pinholes for 3D confocal scanning gives possibility to visualize and analyze nanoobjects with $X Y$ lateral size from $120 \mathrm{~nm} \times 120 \mathrm{~nm}$ and $Z$ height from $10 \mathrm{~nm}$. Using powerful software qualitative and quantitative analysis could be made.

\section{Results}

The sample \#1 was a reference sample without silver nanoparticles. Figure 1 presents 2D image of textile material scanned by CCD camera with $50 \times$ magnification. Figure 2 presents 3D image of textile material scanned by photomultiplier tube (PMT) detectors with $50 \times$ magnification. There are no silver nanoparticles agglomerates observed on the fibres.

The sample $\# 2$ was a reference sample with silver nanoparticles. Figure 3 presents 2D image of textile material scanned by CCD camera with $100 \times$ magnification. 


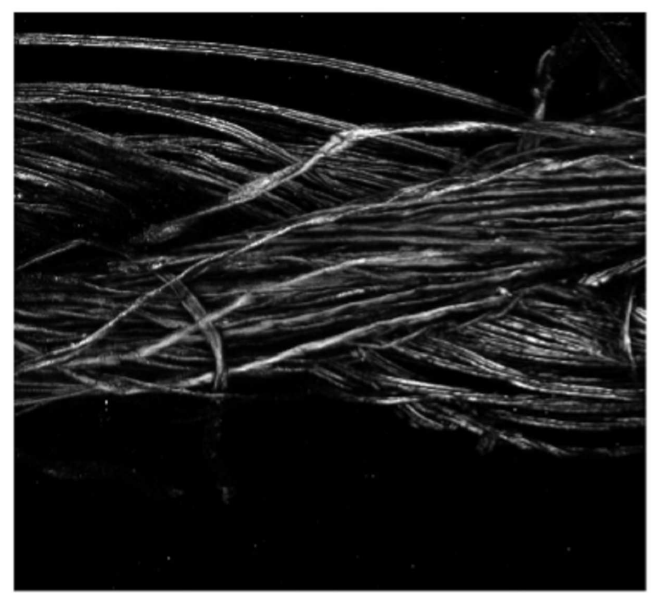

Fig. 1. Presented 2D part of textile material without silver nanoparticles, magnification $50 \times$, detector CCD camera (sample $\# 1$, reference).

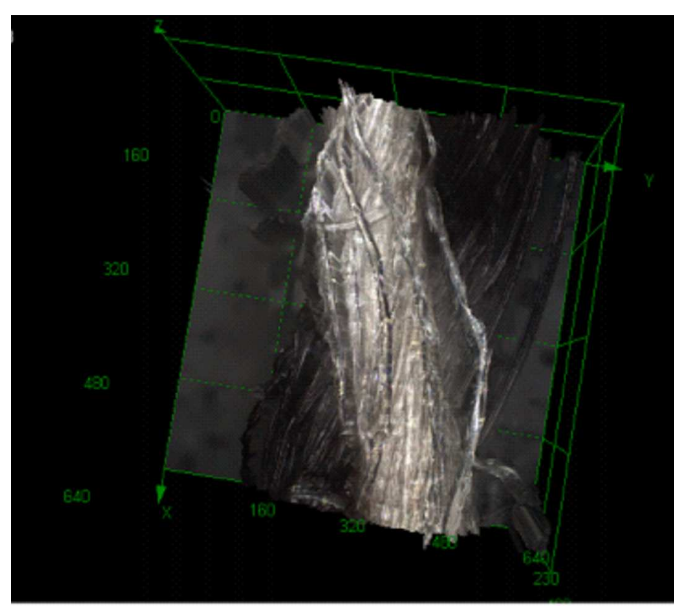

Fig. 2. Presented 3D part of textile material without silver nanoparticles, magnification $50 \times$, detectors - two photomultiplier detectors with double pinholes (sample \#1, reference).

Figure 4 presents 3D image of textile material scanned by PMT detectors with $100 \times$ magnification. There are silver nanoparticles agglomerates observed on the fibres.

As shown above, when silver nanoparticles are present, fibres covered by silver nanoparticles agglomerate randomly and distribution of sizes of agglomerates changes from $200 \mathrm{~nm}$ to $400 \mathrm{~nm}$.

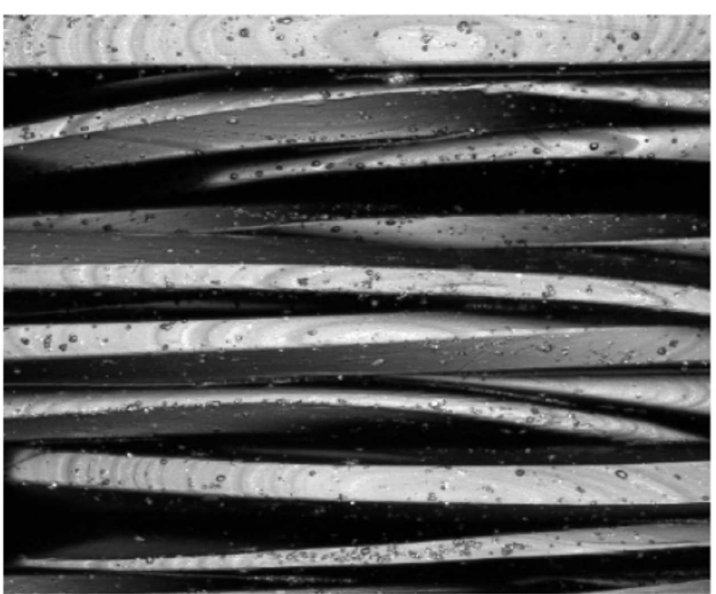

Fig. 3. Presented 2D part of textile material with silver nanoparticles, magnification $100 \times$, detector CCD camera (sample \#2).

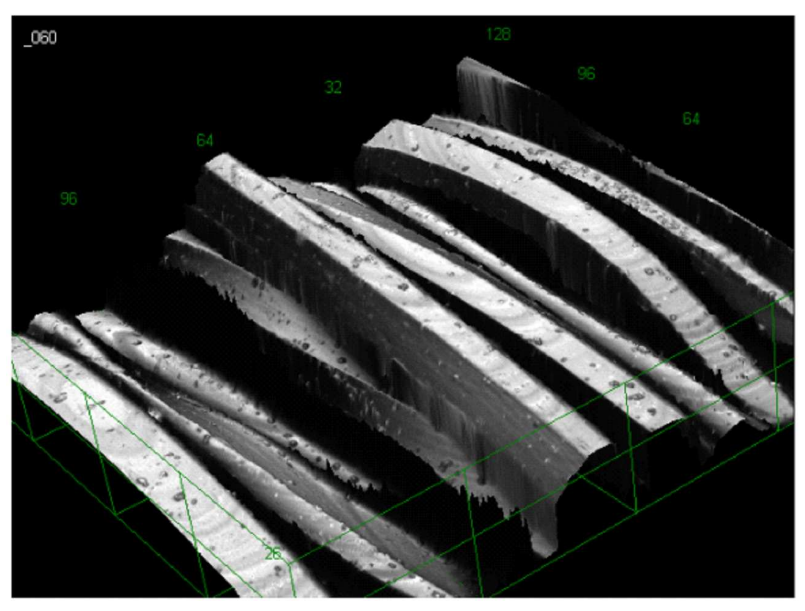

Fig. 4. Presented 3D part of textile material without silver nanoparticles, magnification $100 \times$, detectors - two photomultiplier detectors with double pinholes (sample \#2).

Investigations of "nano-silver" textile material were made quickly, non-destructively and comprehensively with help of Olympus LEXT OLS4000.

For more detailed information, please contact with Dr. Sergey Yatsunenko, Institute of Physics, Polish Academy of Sciences, or visit appropriate website. 